\title{
CT features of pneumonia during COVID-19 pandemic warrant caution: An illustrative case report of cholangiocarcinoma with Pneumocystis jiroveci pneumonia
}

\section{Fang Liu}

Medical School of Chinese PLA; Department of Gastroenterology and Hepatology, the First Medical Center, Chinese PLA General Hospital

Wen Li ( $\sim$ pladr301@sina.com )

Department of Gastroenterology and Hepatology, the First Medical Center, Chinese PLA General Hospital

\section{Case Report}

Keywords: COVID-19 pandemic, chest computed tomography, ground-glass opacities, cholangiocarcinoma, Pneumocystis jiroveci pneumonia

Posted Date: June 4th, 2020

DOI: https://doi.org/10.21203/rs.3.rs-32974/v1

License: (c) (1) This work is licensed under a Creative Commons Attribution 4.0 International License.

Read Full License 


\section{Abstract}

Background: An outbreak of severe acute respiratory syndrome due to coronavirus 2 (SARS-CoV-2) infection (COVID-19) that began at Wuhan, China in December 2019, spreading rapidly across China and many other countries. Considering the high false-negative rate of RT-PCR assay during initial COVID-19 pandemic in China, chest computed tomography (CT) was advocated as a means of corroborating clinically suspected infections.

Case presentation: A 51-year-old man with clinical diagnosis of hepatic portal cholangiocarcinoma and Behcet's disease developed clinical manifestations suggestive of COVID-19 during the pandemic in China. The chest CT showed rapid progression to diffuse ground-glass opacities (GCOs) in both lungs, as in severe cases of confirmed COVID-19. He was finally diagnosed with Pneumocystis jiroveci pneumonia (PJP) according to the medical history, and Caspofungin plus TMP/SMX elicited a rapid response as normalizing both temperature and leukocyte count initially. Unfortunately, dyspnea was aggravated with resumed fever later, and non-invasive ventilation was no longer tenable. The patient himself (with support of his family) declined mechanical ventilatory assistance, succumbing to pneumonia and respiratory failure finally.

Conclusions: CT diagnosis of COVID-19 during the current pandemic should warrant caution.

\section{Background}

By mid-March 2020, spread of SARS-CoV-2 infection (COVID-19) had generally abated in most provinces of China, whereas caseloads outside China were on the rise, signaling a major global outbreak and serious public health emergency ${ }^{1,2}$. Real-time reverse transcription-polymerase chain reaction (RTPCR) is presently deemed the reference standard for definitive diagnosis of COVID-193 , but the high false-negative rate ${ }^{4}$ and general unavailability of this assay initially hampered expeditious screening of infected patients. Radiologic features, especially those of thin-slice chest computed tomography (CT), seemed to help identify early-phase lung infections; and after a fifth trial at Hubei, China ${ }^{5}$, CT studies were advocated to confirm clinically suspected disease. Patients with distinctive clinical manifestations (eg, fever and/or respiratory symptoms, typical chest CT findings, normal and/or low-level leukocyte counts at early stage) were presumed infectious, even if epidemiologic histories were unclear ${ }^{5}$.

Herein, we describe a patient of cholangiocarcinoma with clinical and radiologic manifestations of COVID-19 whose condition was ultimately attributed to Pneumocystis jiroveci pneumonia (PJP). CT interpretations during the current pandemic are thus deserving of caution.

\section{Case Presentation}

A 51-year-old man with gradual worsening of painless jaundice for $>1$ month was admitted on January 17, 2020 to the Department of Gastroenterology and Hepatology at the First Medical Center of PLA 
General Hospital in Beijing, China. Past medical history included vascular replacement (2009) and endovascular repair (2014) of a thoracoabdominal aortic aneurysm. A daily regimen of oral steroid (prednisolone acetate, $10 \mathrm{mg}$ ) and immunosuppressant (azathioprine, $100 \mathrm{mg}$ ) had also been followed for 10 years as treatment of Behcet's disease. At the onset of jaundice, which he alone ascribed to primary biliary cirrhosis, the patient arbitrarily boosted his oral steroid dosage to $60 \mathrm{mg} /$ day.

Upon admission, tapering the high-dose steroid was refused. Serum concentrations of total bilirubin (TBIL, $386.8 \mu \mathrm{mol} / \mathrm{L}$ [peak]), direct bilirubin (DBIL, $344.7 \mu \mathrm{mol} / \mathrm{L}$ [peak]), and CA199 (>20000 $\mu / \mathrm{ml}$ [peak]) were substantially elevated and showed an upward trend. Meanwhile, outcomes of several imaging studies (enhanced abdominal CT, magnetic resonance imaging [MRI], and magnetic resonance cholangiopancreatography [MRCP]) were jointly suggestive of hepatic portal cholangiocarcinoma. On February 7,2020 , the patient underwent percutaneous transhepatic cholangiodrainage, lowering the postprocedural TBIL level to $\sim 100 \mu \mathrm{mol} / \mathrm{L}$. Surgical intervention was anticipated as liver function further improved.

On February 14,2020 , however, a high fever $\left(39.9^{\circ} \mathrm{C}\right)$ developed (Figure 1), without cough, sputum, dyspnea, or other respiratory symptoms. Chest CT performed February 15, 2020 (febrile Day 2) showed inflammation in middle and lower lobes of right lung, with bilateral pleural effusions (Figure 2-A). Leukocyte counts had gradually dwindled, falling below normal values, with similar declines in hemoglobin and platelets (Table 1). The patient denied travel to Wuhan or known contact with confirmed or likely sufferers of COVID-19. In consultation with Respiratory Medicine and the Fever Clinic, COVID-19 was not the primary consideration. A consultant from the Hematology Department raised the possibility of hemophagocytic syndrome due to severe systemic inflammation, but subsequent blood transfusion (February 17, 2 units of suspended erythrocytes) and use of granulocyte stimulating factor were not helpful.

After a 12-day experimental antimicrobial treatment (cefoperazone/sulbactam plus levofloxacin), repeat CT imaging of the chest on February 20 (febrile Day 7, Figure 2-B) and on February 24 (febrile Day 11, Figure 2-C) indicated some resolution of pneumonia and effusions, in support of bacterial infection. Still, high fever persisted almost daily (Figure 1). All cultures (blood x 4, sputum, and bile) and oropharyngeal swabs (influenza $A$ and $B$ ) obtained during this period were negative.

Meanwhile, in chest CT of February 24, slight newly emergent ground-glass opacities (GGOs) were visible in subpleural peripheral distribution (Figure 2-D), and the patient complained of increasing fatigue (Figure 1). An oropharyngeal swab tested for SARS-CoV-2 by real-time RT-PCR proved negative (February 25). Although fungal $\beta$-d-glucan was quite high $(134.9 \mathrm{pg} / \mathrm{ml})$, Aspergillus galactomannan antigen and T-cell spot test for tuberculosis (T-spot. TB; Oxford Immunotec, Abingdon, UK) were negative, as were screenings for human immunodeficiency virus (HIV), Epstein-Barr virus, and cytomegalovirus. Meropenem and voriconazole, given in combination for potential fungal infection, failed to lower the body temperature, which exceeded $39^{\circ} \mathrm{C}$; and the leukocyte count continued to drop, falling to $0.88 \times 10^{9} / \mathrm{L}$ on February 28 (Table 1). 
A chest CT on February 28 (febrile Day 15) showed progression to diffuse GGOs in both lungs (Figure 2$E / F)$, as in patients with severe confirmed COVID-19, and the patient grew increasingly dyspneic (Figure 1). A second oropharyngeal swab for SARS-CoV-2 testing was again negative. Through multidisciplinary consultation with the Fever Clinic and other divisions (Departments of Respiratory Medicine, Medical Imaging, Rheumatology, Cardiovascular Medicine, and Gastroenterology), a consensus diagnosis of PJP (rather than COVID-19) was reached. Voriconazole was then replaced by caspofungin and trimethoprimsulfamethoxazole (TMP/SMX), administered in conjunction with intravenous prednisolone acetate (80 $\mathrm{mg} /$ day). Normal body temperature was restored the evening of February 28 , and the leukocyte count began to normalize. Above therapeutic responses seemed to validate the diagnosis of PJP.

Unfortunately, an exacerbation of dyspnea occurred the night of March 1, 2020, diminishing oxygen saturation. Noninvasive ventilation (NIV) sufficed initially, but fever resumed on March 2, 2020, at which time a third oropharyngeal swab for SARS-CoV-2 tested negative. To address possible co-infections, teicoplanin and ganciclovir were started and soon lowered his body temperature to normal. Cough developed March 4, 2020 (febrile Day 20, Figure 1), and dyspnea was aggravated by a spontaneous pneumothorax. Chest radiographs taken separately on March 4 and March 5, 2020 continued to show diffuse interstitial opacities and consolidation. Non-invasive ventilation was no longer tenable, but the patient himself (with support of his family) declined mechanical ventilatory assistance, succumbing to pneumonia and respiratory failure on March 7, 2020.

\section{Discussion And Conclusion}

In this patient with a clinical diagnosis of hepatic portal cholangiocarcinoma, there was a looming possibility of COVID-19. His condition was further complicated by 10 years of dual-agent (prednisolone acetate plus azathioprine) immunosuppression for Behcet's disease, including an arbitrary boost in steroid dosage $(60 \mathrm{mg} /$ day $)>1$ month before in-hospital fever erupted. That being the most common symptom of COVID-196, we acted promptly to establish a diagnosis and curtail potential spread. The patient had no clear epidemiologic link, and his initial chest CT suggested bacterial pneumonia. He responded well to antibiotics, excluding COVID-19 at this juncture. However, the fever persisted $\left(>39^{\circ} \mathrm{C}\right)$, and peripheral lung GGOs emerged on CT scan (February 24, 2020), causing alarm and compelling RTPCR testing for SARS-CoV-2. The latter was negative, but fungal infection was evident, based on a high level of $\beta$-d-glucan. Even so, meropenem and voriconazole treatment was ineffective.

The chest CT showed rapid progression to diffuse GGOs in both lungs (February 28, 2020, febrile Day 15), as in severe cases of confirmed COVID $-19^{7}$, and dyspnea was increasingly problematic. Leukocyte counts also dwindled to below-normal levels. The above were commonly exhibited by patients in Wuhan, China ${ }^{5}$ and demanded a firm diagnosis as a matter of urgency. Oropharyngeal swabs were tested on two more occasions for SARS-CoV-2, with negative results. However, the false-negative rate of RT-PCR assay is high ${ }^{4}$, and a certain percentage $(25.9 \%)$ of infected patients living outside Wuhan had no clear points of contact ${ }^{6}$, so COVID-19 remained a possibility. 
Our multidisciplinary panel offered a consensus diagnosis of PJP (rather than COVID-19) based on the following evidence: (1) The patient had no clear epidemiologic exposure, and the 27-day hospitalization prior to onset of fever exceeded the longest known incubation period of COVID-196; (2) human-to-human transmissibility of SARS-CoV-2 is high through droplets or direct contact ${ }^{6,8,9}$, yet no family members, patients, or medical staff had fevers or respiratory symptoms; (3) the patient's immunocompromised state and comorbid conditions (cholangiocarcinoma, Behcet's disease) heightened his vulnerability to PJP; (4) chest CT (February 28, 2020) showed diffuse, symmetric GGOs (largely perihilar), more consistent with PJP' ${ }^{10}$, whereas diffuse subpleural GGOs are typical of COVID-197; and (5) $\beta$-d-glucan elevation was compatible with PJP ${ }^{11}$. As it happened, the first-line regimen for non-HIV patients with PJP (ie, caspofungin plus TMP/SMX) ${ }^{12}$, elicited a rapid response, normalizing both temperature and leukocyte count.

PJP is a potentially life-threatening infection in immunocompromised patients, inflicting high morbidity and mortality. In the absence (vs presence) of HIV infection, such patients deteriorate rapidly, showing greater risk of respiratory failure and death ${ }^{13-14}$. Because microbial confirmation is often not feasible or difficult to obtain, high-resolution CT is the chief means of guiding clinical diagnoses when appropriate ${ }^{10}$. Had the pneumonia resolved, the patient's cancer prognosis was still grim. He and his family understandably refused mechanical ventilation, bronchoalveolar lavage for PCR testing, and autopsy. We fully respected their wishes, despite the lingering void in pathologic and culture confirmation.

The addition of chest CT for diagnosis of COVID-19 created a sharp increase and peak in confirmed new cases reported on February 12, 2020 in China. We have actually shared this patient's chest CT in remote consultation with colleagues in Wuhan, who believe this is a severe case of COVID-19. On the other hand, our diagnosis of PJP is rooted in multidisciplinary deliberation, relying heavily on medical history and the hospital course. Clearly, a comprehensive analysis is preferable to blind interpretation of CT findings. It may be that the role of chest CT during this pandemic has been overstated in haste and should be re-examined going forward to avoid misdiagnosis, unnecessary panic, and wasted medical resources.

\section{Abbreviations}

SARS-CoV-2: severe acute respiratory syndrome due to coronavirus 2

COVID-19: SARS-CoV-2) infection

RT-PCR: real-time reverse transcription-polymerase chain reaction

CT: computed tomography

PJP: Pneumocystis jiroveci pneumonia

TBIL: total bilirubin 
DBIL: direct bilirubin

MRI: magnetic resonance imaging

MRCP: magnetic resonance cholangiopancreatography

GGOs: ground-glass opacities

T-spot. TB: T-cell spot test for tuberculosis

HIV: human immunodeficiency virus

TMP/SMX: trimethoprim-sulfamethoxazole

NIV: noninvasive ventilation

\section{Declarations}

Ethics approval and consent to participate: The patient agreed to requisite examinations and signed informed consent prior to invasive treatments. The study was approved by the Ethics Committee of the PLA General Hospital.

Consent for publication: The deceased patient's next of kin had provided permission and written consent for publication of medical records and images about their deceased relative in publications.

Availability of data and materials: All data generated or analysed during this study are included in this published article [and its supplementary information files].

Competing interests: The authors declare that they have no competing interests.

Funding: No funding.

Author Contributions: Fang Liu and Wen Li participated in the patient hospitalization management. Fang Liu performed the data analyses and wrote the manuscript. Wen Li designed the work that led to the submission and revised the manuscript. All authors have read and approved the final manuscript.

Acknowledgements: Not applicable

\section{References}

1. Chen N, Zhou M, Dong X, et al. Epidemiological and clinical characteristics of 99 cases of 2019 novel coronavirus pneumonia in Wuhan, China: a descriptive study. Lancet 2020;395:507-513..

2. Zhu N, Zhang D, Wang W, et al. A Novel Coronavirus from Patients with Pneumonia in China, 2019. N Engl J Med 2020;382:727-733. 
3. Corman VM, Landt O, Kaiser M, et al. Detection of 2019 novel coronavirus (2019-nCoV) by real-time RT-PCR. Euro Surveill 2020;25.

4. Chan JF, Yuan S, Kok KH, et al. A familial cluster of pneumonia associated with the 2019 novel coronavirus indicating person-to-person transmission: a study of a family cluster. Lancet 2020;395:514-523.

5. General Office of National Health Committee. Office of state administration of traditional Chinese medicine. Notice on the issuance of a programme for the diagnosis and treatment of novel coronavirus (2019-nCoV) infected pneumonia (trial fifth edition) (2020-02-09) [EB/OL]. http://bgs.satcm.gov.cn/zhengcewenjian/2020-02-09/12929.html

6. Guan WJ, Ni ZY, Hu Y, et al. Clinical Characteristics of Coronavirus Disease 2019 in China. N Engl J Med 2020 Feb 28.

7. Zu ZY, Jiang MD, Xu PP, et al. Coronavirus Disease 2019 (COVID-19): A Perspective from China. Radiology 2020 Feb 21:200490.

8. Li Q, Guan X, Wu P, Wang X, et al. Early transmission dynamics in Wuhan, China, of novel coronavirus-infected pneumonia. N Engl J Med 2020 Jan 29 [Epub ahead of print].

9. Wang D, Hu B, Hu C, et al. Clinical characteristics of 138 hospitalized patients with 2019 novel coronavirus-infected pneumonia in Wuhan, China. JAMA 2020 Feb 7 [Epub ahead of print].

10. Cereser L, Dallorto A, Candoni A, et al. Pneumocystis jirovecii pneumonia at chest High-resolution Computed Tomography (HRCT) in non-HIV immunocompromised patients: Spectrum of findings and mimickers. Eur J Radiol 2019;116:116-127.

11. Morjaria S, Frame A, Franco-Garcia A,et al. Clinical performance of $(1,3)$ beta-D glucan for the diagnosis of Pneumocystis pneumonia (PCP) in cancer patients tested with PCP polymerase chain reaction. Clin Infect Dis 2019. 69: 1303-1309.

12. Maschmeyer G, Helweg-Larsen J, Pagano L, et al. ECIL guidelines for treatment of Pneumocystis jirovecii pneumonia in non-HIV-infected haematology patients. J Antimicrob Chemother 2016;71:2405-2413.

13. Roux A, Canet E, Valade $S$, et al. Pneumocystis jirovecii pneumonia in patients with or without AIDS, France. Emerging Infect Dis 2014; 20:1490-1497.

14. Kato H, Samukawa S, Takahashi H, et al. Diagnosis and treatment of Pneumocystis jirovecii pneumonia in HIV-infected or non-HIV-infected patients-difficulties in diagnosis and adverse effects of trimethoprim-sulfamethoxazole. J Infect Chemother 2019;25:920-924.

\section{Table}

Due to technical limitations, table 1 is only available as a download in the supplemental files section.

\section{Figures}




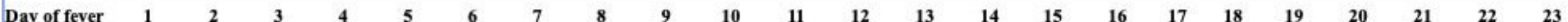

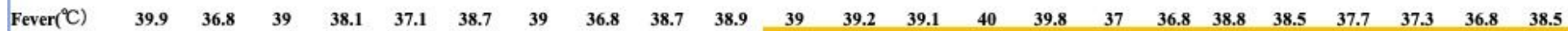

Fatigue

Dyspnea

Cough

Antibiotics

levofloxacin+cefoperazone sodium/sulbactam sodium

Meropenem

Voriconazole

Caspofungin +trimethoprim-sulfamethoxazole (TMP/SMX)

teicoplanin + ganciclovir

Figure 1

Symptoms, Maximum Body Temperatures and Antibiotics Application According to Day of Fever
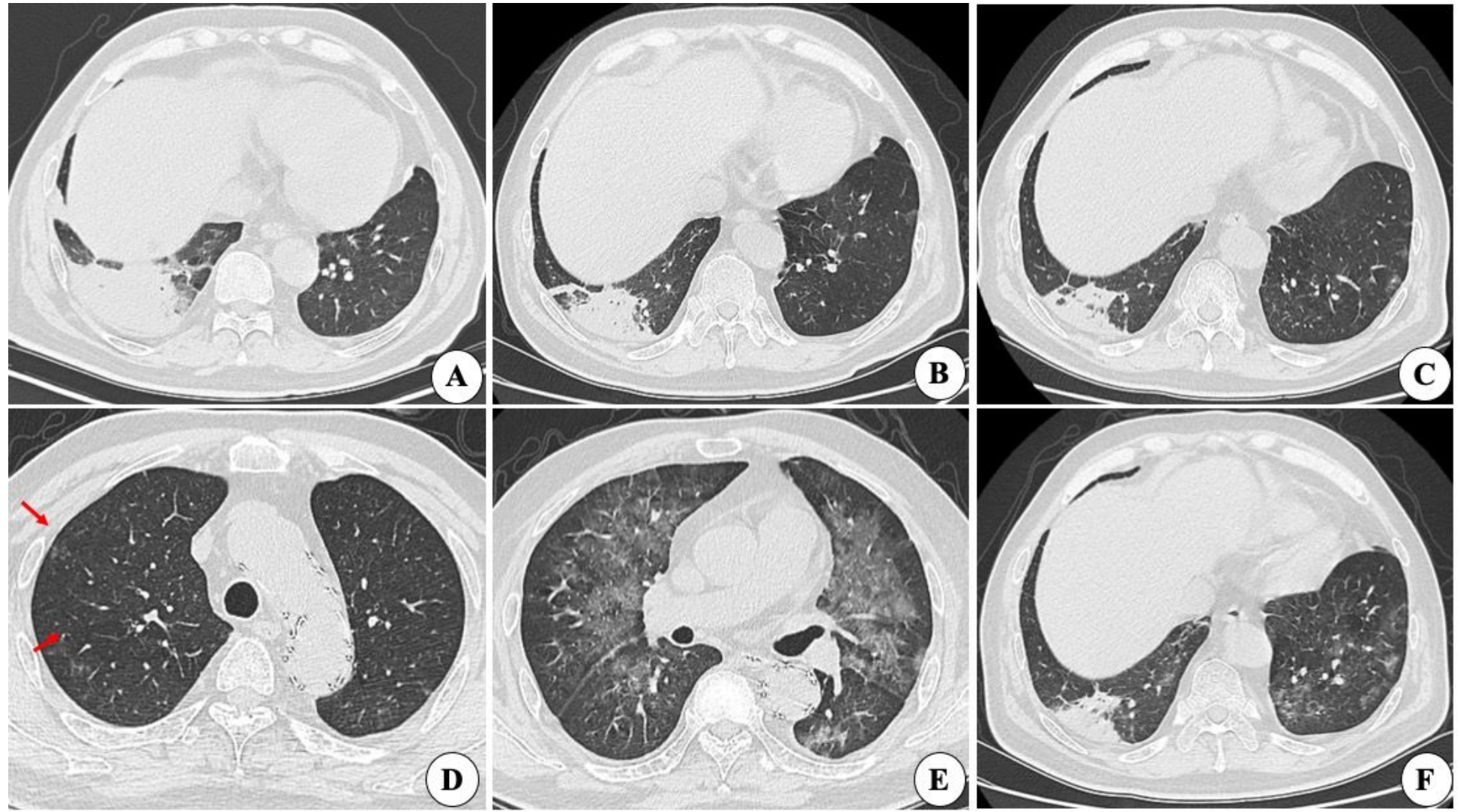

Figure 2 Changes of Pneumonia, pleural effusion and GGOs in chest CT imaging

A, February 15, 2020 (febrile day 2), inflammation in middle and lower lobes of right lung, with bilateral pleural effusions

B. February 20, 2020 (febrile day 7), some resolution of pneumonia and effusions

C. February 24, 2020 (febrile day 11), further resolution of pneumonia and effusions

D. February 24, 2020 (febrile day 11), slight newly emergent GGOs in subpleural peripherally distribution (red arrow)

E-F. February 28, 2020 (febrile day 15), progressive diffuse GGOs in both lungs and further resolution of pneumonia and effusions(F)

\section{Figure 2}




\section{Supplementary Files}

This is a list of supplementary files associated with this preprint. Click to download.

- Table1.jpg 